\title{
Functional Significance of Erythropoietin Receptor Expression in Breast Cancer
}

\author{
Murat O. Arcasoy, Khalid Amin, Aysen F. Karayal, Shu-Chuan Chou, \\ James A. Raleigh, Mahesh A. Varia, and Zishan A. Haroon \\ Department of Medicine (MOA, AFK), Duke University Medical Center, Durham, and Department of Radiation \\ Oncology (S-CC, JAR, MAV), University of North Carolina School of Medicine, Chapel Hill, North Carolina; and SRI \\ International (KA, ZAH), Menlo Park, California
}

\begin{abstract}
SUMMARY: Erythropoietin (EPO) is the principal hematopoietic cytokine that regulates mammalian erythropoiesis by binding to its transmembrane receptor EpoR. Recent experimental evidence suggests that the biologic effects of EPO are not limited to the regulation of erythropoiesis. In studies focusing on nonhematopoietic effects of EpoR signaling, we found high levels of EpoR protein expression in human breast cancer cells. The purpose of the present study was to evaluate clinical breast cancer specimens for EPO and EpoR expression, characterize the relationship between EPO expression and tumor hypoxia in biopsies prelabeled with hypoxia marker pimonidazole, analyze breast cancer cell lines for EpoR expression, and study the functional significance of EpoR expression in breast cancer cells in vivo. Immunohistochemical analysis for EPO, EpoR expression, and pimonidazole adducts was performed on 26 tumor biopsies with contiguous sections from 10 patients with breast cancer. High levels of EpoR expression were found in cancer cells in $90 \%$ of tumors. EPO expression was found in $60 \%$ of tumors and EPO and EpoR colocalization in tumor cells was present in many cases. The expression pattern of EPO with respect to tumor hypoxia was variable, without consistent colocalization of EPO and hypoxia in tumor cells. Human and rat breast cancer tissue culture cells express EpoR mRNA and protein. To study the in vivo function of EpoR expression in breast cancer cells, we used rat syngeneic R3230Ac mammary adenocarcinoma cells in a tumor Z-chamber model (dual porous plexiglass chambers containing fibrin gel, cancer cells, and a putative anti-tumor compound implanted into the subcutaneous tissue of rats). Local, one-time administration of a neutralizing anti-EPO antibody, soluble EPO receptor, or an inhibitor of Jak2, a cytoplasmic tyrosine kinase essential for EPO-mediated mitogenesis, resulted in a delay in tumor growth with $45 \%$ reduction in maximal tumor depth in tumor Z-chambers in a dose-dependent manner. These studies demonstrate the expression of functional receptors for EPO in breast cancer cells. (Lab Invest 2002, 82:911-918).
\end{abstract}

$F$ rythropoietin (EPO) is a glycoprotein hormone $C$ that is essential for the proliferation, viability, and terminal differentiation of erythroid progenitor cells (Krantz, 1991; Lin et al, 1996; Wu et al, 1995). The biologic effects of EPO are mediated by its interaction with its specific transmembrane receptor, EpoR, expressed on the surface of erythroid progenitor cells (D'Andrea et al, 1989; Lodish et al, 1995; Youssoufian et al, 1993). EpoR is a member of the type I cytokine receptor family, characterized by a lack of intrinsic tyrosine kinase activity (Bazan, 1990; D'Andrea et al, 1990; Klingmüller, 1997; Wojchowski et al, 1999). The binding of EPO to its cognate receptor results in a ligand-induced conformational change of receptor dimers that allows the tyrosine phosphorylation of Jak2 (Livnah et al, 1999; Remy et al, 1999; Witthuhn et

DOI: 10.1097/01.LAB.0000020415.72863.40

Received February 28, 2002.

This work was supported by grants from the National Institutes of Health DK-02566 (MOA), CA68826 (JAR, MAV), and RR00046, Department of Defense BC-962506 (MAV, JAR), and the State of North Carolina.

Address reprint requests to: Dr. Zishan A. Haroon, Director, In Vivo Biology Program, SRI International, 333 Ravenswood Avenue, Menlo Park, CA 94025. E-mail: zishan.haroon@sri.com al, 1993), a receptor-associated cytoplasmic tyrosine kinase that is essential for EpoR-mediated mitogenesis and prevention of apoptosis (Zhuang et al, 1995; Zhuang et al, 1994).

Expression of functional receptors for EPO has been reported in several nonhematopoietic cell types, including vascular endothelial cells (Anagnostou et al, 1994), neuronal cells (Masuda et al, 1993), myoblasts (Ogilvie et al, 2000), and kidney (Westenfelder et al, 1999). Recent studies have provided evidence for nonhematopoietic functions for EpoR signaling. For instance, inactivation of EPO or EpoR function in mice by targeted gene disruption was associated with defects in cardiac morphogenesis and abnormalities in the vascular network (Wu et al, 1999). In myoblasts, functional EpoR signaling was associated with proliferation and decreased terminal differentiation to form myotubules (Ogilvie et al, 2000). In brain, expression of EPO receptors in neurons has been shown to confer an EPO-mediated neuroprotective effect during glutamate or ischemia-induced injury in vivo (Brines et al, 2000; Digicaylioglu and Lipton, 2001; Morishita et al, 1997; Sakanaka et al, 1998).

EPO is normally produced in the fetal liver and adult kidney, where hypoxia is the major stimulus for EPO expression (Bunn and Poyton, 1996; Lacombe and 
Mayeux, 1998). More recently, estrogen-dependent EPO production was demonstrated in the female reproductive tract, specifically in the uterus (Yasuda et al, 1998) and oviduct (Masuda et al, 2000). Another study reported EPO expression in lactating breast (Juul et al, 2000). In our studies investigating nonhematopoietic functions of EpoR signaling, we asked whether breast cancer cells express EPO protein. We carried out immunohistochemical analysis of primary breast cancer specimens and found high levels of EPO expression in cancer cells in $60 \%$ of tumor biopsies. Surprisingly, EpoR protein expression was observed not only in tumor vessels, but also in tumor cells in $90 \%$ of biopsies. In further studies, we evaluated the pattern of EPO expression with respect to cellular hypoxia in tumor cells, studied monolayer cultures of breast cancer cell lines for EpoR expression, and investigated the functional significance of EpoR expression in breast cancer cells in an in vivo model.

\section{Results \\ Expression of EPO and EpoR in Clinical Breast Cancer Specimens}

We studied a total of 26 biopsies from 10 primary breast cancer clinical specimens by immunohistochemical analysis. Multiple biopsies were obtained from each quadrant of the tumors and contiguous tissue sections were evaluated for EPO, EpoR expression, and the presence of immunoreactivity for pimonidazole adducts as a marker for cellular hypoxia (Raleigh et al, 1998). Figure 1 illustrates representative photomicrographs of immunostaining results. Of 10 primary tumors, only one showed no EpoR immunoreactivity in three separate biopsies. Strong EpoR immunoreactivity in tumor cells was present in 21 of 26 biopsies (Fig. 1, A, D, and F). EpoR expression was also detected in endothelial cells lining tumor vessels and, notably, lack of EpoR immunoreactivity was observed in surrounding normal mammary ducts (data
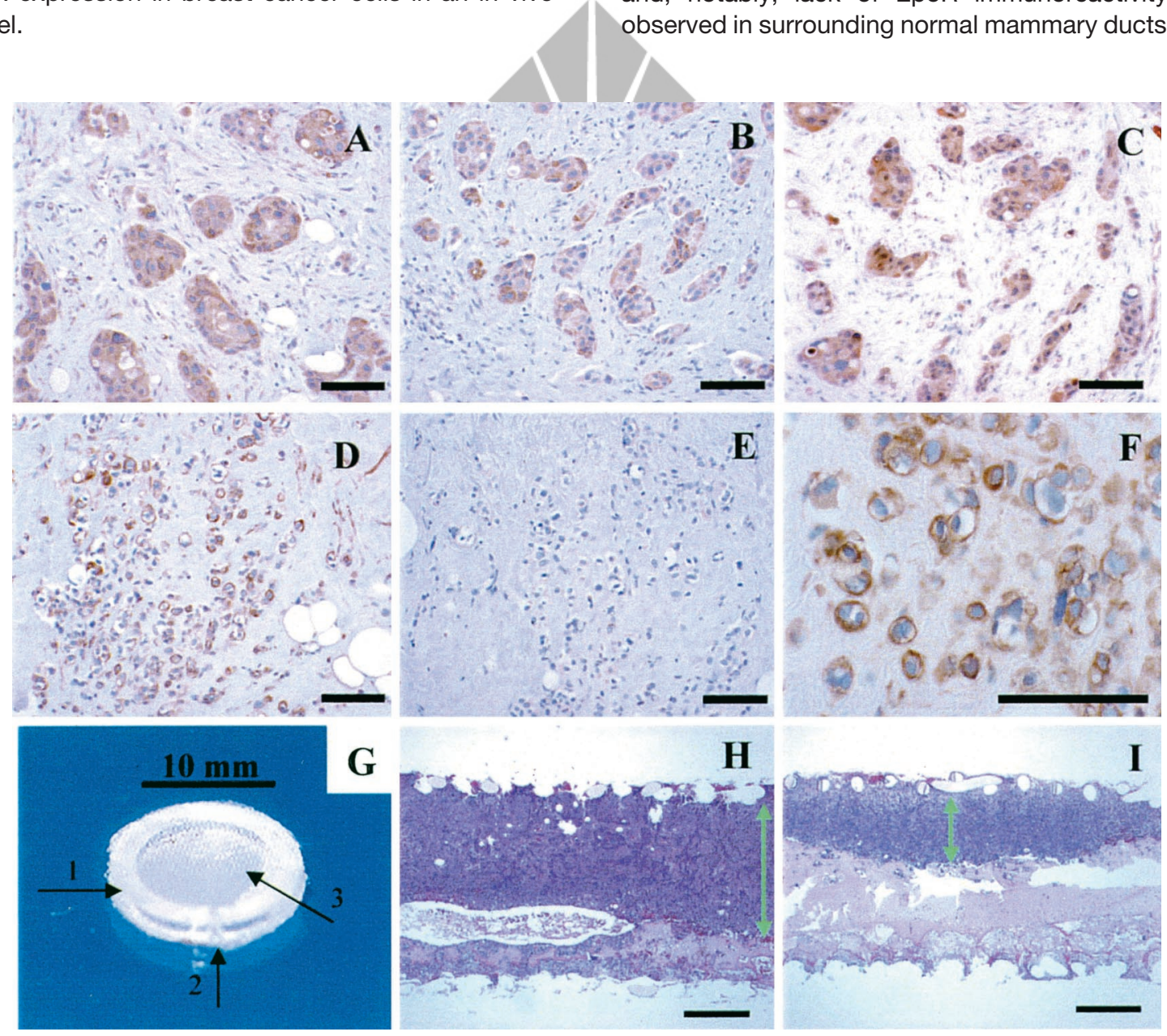

Figure 1.

Results of immunohistochemical studies. Contiguous tissue sections from breast tumors were immunostained to detect EPO, EpoR, or presence of pimonidazole adducts as a marker for hypoxia. Panels A, B, and C, Immunostaining of contiguous sections from the same tumor showing reactivity for EpoR (A), EPO (B), and pimonidazole adducts (C). Panels D and E, Immunostaining of contiguous sections for EpoR (D) and EPO (E), demonstrating EpoR immunoreactivity and no EPO expression. Panel F, High magnification of tissue section showing strong EpoR immunoreactivity in breast cancer cells. Panel G, Z-chamber consists of plexiglass rings (1), with an access port on the side (2), and covered with $180 \mu \mathrm{m}$ nylon mesh on both sides allowing the in-growth of vessels (3). Panels $\mathrm{H}$ and I, Representative section of tumor removed from Z-chamber stained with hematoxylin and eosin $(\mathrm{H} \& \mathrm{E})$. Panel H, Histology of typical tumor formation at Day 7 after implantation of the T-ZCs. The maximal tumor depth (green arrow) is representative of negative control chambers containing rat mammary adenocarcinoma R3230 Ac cells and vehicle. Panel I, Reduction in maximal tumor depth (green arrow) observed in a T-ZC containing recombinant soluble EpoR (compare panels $\mathrm{H}$ and I). A to F, bar indicates $100 \mu \mathrm{m} ; \mathrm{H}$ and I, bar indicates $500 \mu \mathrm{m}$. 
not shown). EPO expression was observed in 6 of the 10 primary tumor specimens and in 13 of 26 tumor biopsies (Fig. 1B). EPO immunoreactivity was not observed in the normal ducts adjacent to the breast cancer cells (data not shown). Examination of contiguous sections in each case allowed the determination of colocalization of EPO and EpoR immunoreactivity. In tumor cells, EpoR expression colocalized with EPO immunoreactivity in $92 \%$ of the 13 biopsies that were strongly positive for EPO expression (compare Fig. 1, $A$ and B). Colocalization of EPO expression was not observed in 8 biopsies with EpoR immunoreactivity (compare Fig. 1, D and E). A summary of results of immunostaining of the 26 breast cancer biopsies is illustrated in Table 1. The data set illustrates positive immunoreactivity for EPO, EpoR, and pimonidazole adducts (hypoxia) and colocalization of expression in contuguous tissue sections.

\section{EPO Expression and Tumor Hypoxia}

The principal site of EPO production in adults is the kidney where EPO gene expression is stimulated by hypoxia (Lacombe and Mayeux, 1998). In the next set of experiments, we wished to determine whether EPO expression in breast cancer cells correlated with the presence of tumor hypoxia. Labeling of tumor hypoxia by pimonidazole hydrochloride administration to the patients was carried out as described previously (Raleigh et al, 1998). Contiguous tissue sections from the same tumor biopsies were immunostained for EPO, EpoR, and pimonidazole adducts as an immunohistochemical marker for cellular hypoxia. Positive immunoreactivity for pimonidazole adducts in tumor cells was present in 16 of 26 biopsies (Table 1). A representative photomicrograph demonstrating a region of hypoxic tumor cells is illustrated in Figure 1C. As summarized in Table 1, colocalization of EPO expression in hypoxic tumor regions in contiguous sections was present in 7 of the 16 (44\%) tumor biopsies in which cellular hypoxia was detected (compare Fig. 1, $B$ and $C$ ). The presence or absence of hypoxia in tumor cells did not necessarily translate into a distinct expression pattern for EPO. For instance, our data shows lack of EPO colocalization in contiguous sections in 9 of 16 tumors with cellular hypoxia (Table 1). Despite a small sample number, these findings suggest an inconsistent relationship between cellular hypoxia and EPO expression in breast tumors and possi-

Table 1. Results of Immunohistochemical Studies of Breast Cancer Biopsies

\begin{tabular}{lc}
\hline \multicolumn{1}{c}{ Antigen } & Immunoreactivity $(n=26)$ \\
\hline Epo & 13 \\
EpoR & 21 \\
Hypoxia & 16 \\
Epo + EpoR & 12 \\
Epo + hypoxia & 07 \\
EpoR + hypoxia & 11 \\
\hline
\end{tabular}

EPO, erythropoietin; EpoR, erythropoietin receptor. bly indicates the presence of other factors that regulate EPO expression in breast cancer cells.

\section{EPO Receptor Expression in Human Breast Cancer Cell Lines}

We then investigated for EpoR expression in human breast cancer tissue culture cell lines that may be used to generate models for studies of the function of EpoR signaling in breast cancer. The expression of EpoR mRNA transcripts and protein in breast cancer cell lines was examined. As illustrated in Figure 2, we performed RT-PCR using three different oligonucleotide primer sets specific for EpoR (Fig. 2A). Figure 2B illustrates the presence of specific EpoR mRNA fragments using two different oligonucleotide primer sets in mammary carcinoma MCF-7, MDA-MB231, and
A

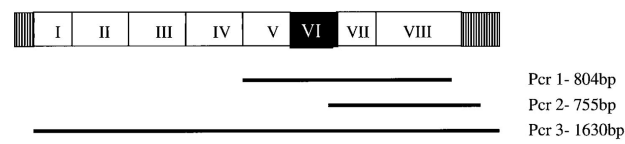

B
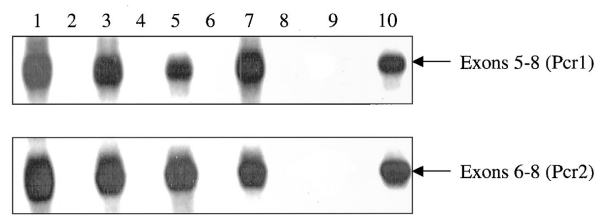

$\mathrm{C}$
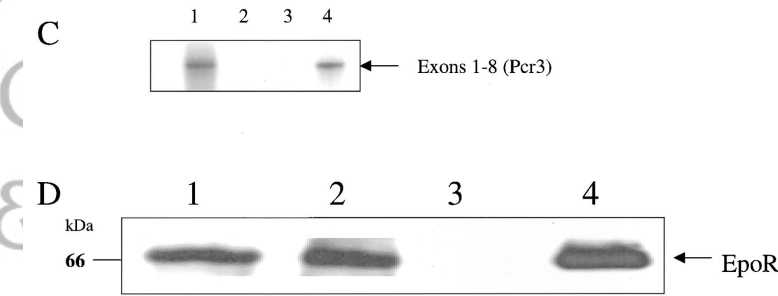

Figure 2.

Expression of EpoR mRNA and protein in breast cancer cells. A, Diagram of EpoR gene coding sequence. The eight exons of EpoR coding sequence are numbered. Exon 6 encodes the transmembrane region and hatched boxes indicate untranslated regions. The PCR amplification fragments obtained with three different sets of primers and the expected amplification product sizes are shown. B, Expression of EpoR mRNA transcripts in breast cancer cells. Total RNA was isolated from breast cancer cells and RT-PCR was performed to amplify exons 5 to 8 (upper panel) and exons 6 to 8 (lower panel) of EpoR using two different oligonucleotide primer sets. The PCR products were separated in $1 \%$ agarose gels and Southern blotting was followed by hybridization to ${ }^{32} \mathrm{PdCTP}$-labeled full-length EpoR cDNA probe. The arrows indicate 804-bp EpoR transcript (Pcr 1) and 755-bp transcript (Pcr2) in the upper and lower panels, respectively. Lanes 1 and 2: MCF-7 cells; Lanes 3 and 4: MDA-MB-231; Lanes 5 and 6: SK-BR3; Lanes 7 and 8: Primary human breast cancer. Lanes 1, 3, 5, and 7 are RT-PCR. Lanes 2, 4, 6, and 8 are negative control PCRs without RT. Lane 9, negative control PCR with water. Lane 10, positive control PCR using plasmid containing full-length EpoR cDNA. C, Expression of full-length EpoR mRNA transcript in MCF-7 cells. RT-PCR amplification of entire coding region of EpoR in MCF-7 cells. Lane 1, MCF-7 cDNA amplification. Lane 2, no RT negative control. Lane 3, water negative control. Lane 4, positive control PCR using EpoR cDNA plasmid. D, Expression of EpoR protein in human breast cancer cell lines. Total cell lysates were subjected to immunoprecipitation as described in "Materials and Methods" and immunoblotting was carried out using anti-EpoR C-20 antibody. Lane 1, MDA-MB231 cells. Lane 2, MCF-7 cells. Lane 3, Negative control 32D cells. Lane 4, Positive control, 32D cells transfected with EpoR expression vector. The molecular weight marker is shown $66 \mathrm{kd}$. The arrow indicates the position of EpoR protein. 
SK-BR3 cells as well as a primary breast carcinoma RNA specimen (Lanes 1, 3, 5, and 7, respectively). No PCR amplification products were observed in negative control samples for each PCR reaction without RT (Lanes 2, 4, 6, and 8), as well as water negative control (Lane 9). Figure 2C demonstrates the presence of 1.6-kb EpoR mRNA transcript in MCF-7 cells containing the entire coding region (Lane 1). The PCR products were subcloned and sequenced, confirming the presence of EpoR cDNA sequences. We then investigated for EpoR protein expression in human breast cancer cell lines by immunoprecipitation using polyclonal anti-human EpoR antibody followed by Western analysis and immunoblotting. Figure 2D illustrates results of a representative Western blot demonstrating the presence of 66-kd EpoR reactivity in EpoR immunoprecipitates of MDA-MB231 and MCF-7 cells (Lanes 1 and 2, respectively), comparable to the positive control sample (Lane 4) from myeloid 32D cells transfected with EpoR expression vector (Arcasoy et al, 1997). No EpoR protein was detected in immunoprecipitates of negative control cell lysates (Lane 3). In other experiments, we also confirmed the cell-surface expression of EpoR using biotinylated EPO and flow cytometry in MCF-7 and MDA-MB231 cells (not shown).

\section{Inhibition of Tumor Growth by EPO-EpoR Antagonists In Vivo}

In the next set of experiments, we investigated the functional significance of EpoR expression in breast cancer cells with respect to tumor growth. We used rat syngeneic R3230Ac mammary adenocarcinoma cell line in a tumor Z-chamber (T-ZC) model (Fig. 1G), described in detail in "Materials and Methods." Briefly, in this model, the cancer cells are suspended in a solution of fibrinogen, injected into T-ZCs along with thrombin and putative anti-tumor compounds, implanted into the subcutaneous tissue of rats, and harvested in 1 to 2 weeks. These dual-porous T-ZC containing fibrin gel allow the in-growth of vessels and development of tumor tissue that can be later quantified by assessment of depth of tumor tissue inside the T-ZC.

First we confirmed the expression of EpoR protein in rat R3230Ac mammary adenocarcinoma cells by anti-EpoR immunoprecipitation and immunoblotting (not shown). We then tested the in vivo anti-tumor activity of three different inhibitors of EPO-EpoR function: recombinant soluble EpoR (sEpoR), a neutralizing monoclonal anti-EPO antibody (MAB-287), and a specific inhibitor of Jak2 tyrosine kinase activity tyrphostin B42 (AG490) administered into the T-ZC (Bright et al, 1999; Meydan et al, 1996; Nielsen et al, 1997). In a previous study of prolactin-induced Jak2 activation in breast cancer cells, AG490 was reported to inhibit proliferation of breast cancer cell lines in vitro (Yamauchi et al, 2000). Negative control T-ZC included PBS or dimethyl sulfoxide (DMSO) alone and there was no difference in the depth of tumor tissue between the two different vehicles. Representative photomicro- graphs demonstrating histologic analysis of $\mathrm{T}-\mathrm{ZC}$ are illustrated in Figure 1, H and I. Quantitative analysis of maximum tumor depth was carried out for all treatment groups and expressed as the percentage of tumor depth compared with control group (100\%). Figure 3 illustrates the summary of quantitative results of the in vivo experiments in which three different doses of each compound were tested and the tumor chambers were harvested on Day 7 after implantation. One-time, intrachamber administration of three increasing doses of sEpoR, ranging from 1.25 to 20 $\mu \mathrm{g} / \mathrm{ml}$, resulted in a $25 \%$ to $45 \%$ reduction in tumor depth compared with controls in a dose-dependent manner (Fig. 3). The treatment effect compared with controls was significant for the medium and highest doses $(p=0.01$ and $p=0.001$, respectively). Local administration into T-ZC of neutralizing anti-EPO antibody (MAB-287) ranging from 10 to $200 \mu \mathrm{g} / \mathrm{ml}$ was also associated with delay in tumor growth in a dose-dependent fashion (Fig. 3). The lowest concentration of anti-EPO antibody resulted in a $22 \%$ reduction of tumor depth compared with control $(p=0.07)$, whereas in the presence of 100 and $200 \mu \mathrm{g} / \mathrm{ml}$ of antibody, there was a significant $27 \%$ and $44 \%$ decrease in tumor depth compared with controls $(p=$ 0.02 and $p=0.0003$, respectively). As illustrated in Figure 3, the administration of AG490 into T-ZCs also resulted in delay in tumor growth. Compared with controls, there was a significant $29 \%$ to $45 \%$ reduction in tumor depth at all three doses tested, ranging from 20 to $200 \mu \mathrm{g} / \mathrm{ml}(p=0.01, p=0.03$, and $p=$ 0.0006, respectively). Taken together, these results indicate that one-time, local administration of sEpoR, anti-EPO monoclonal antibody or AG490 results in a

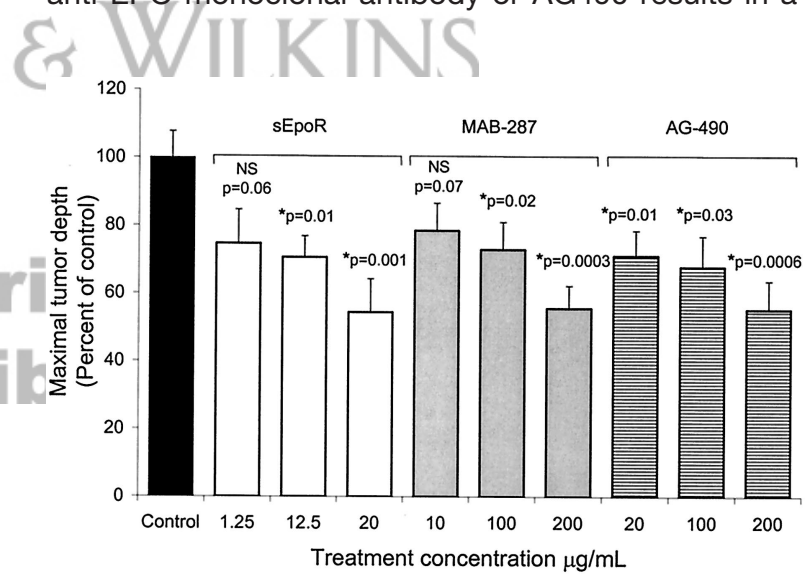

Figure 3.

Inhibition of tumor growth by EPO-EpoR antagonists. Rat R3230Ac mammary adenocarcinoma cells were injected into tumor Z-chambers as described in "Materials and Methods." The chambers contained one-time doses each of recombinant sEpoR, MAB-287 anti-EPO neutralizing antibody, AG-490 Jak2 tyrosine kinase inhibitor, or vehicle (Control). Treatment groups are indicated on top of the chart and final concentrations of each compound are indicated on the $x$ axis. At Day 7, the tumors in the Z-chambers were removed and H\&E staining was performed. Quantitative analysis of maximal tumor depth was carried out in each treatment group and is expressed as a percentage of control, as indicated in the $y$ axis. The data is expressed as mean \pm standard error of the mean. To determine significant effects of treatment, the mean of each treatment group was compared with control using the Students $t$ test (two-tail) and $p$ values for each comparison are indicated above each bar. ${ }^{*} p$ $<0.05$. NS, not significant. 
significant $45 \%$ reduction in tumor depth in the T-ZC model in a dose-dependent manner.

\section{Discussion}

We report the expression of functional receptors for EPO in breast cancer cells and that administration of EpoR signaling antagonists is associated with tumor growth inhibition in vivo. A series of recent studies have demonstrated that EPO has diverse biologic effects that are not limited to the regulation of erythropoiesis. Expression of EPO receptors in several nonhematopoietic cell types, such as vascular endothelial cells, has been associated with the discovery of novel physiologic effects of EPO in nonhematopoietic tissues. For instance, in the developing heart, EPO and EpoR function is required for normal cardiac morphogenesis (Wu et al, 1999). In the female reproductive tract, a paracrine EPO-EpoR system has been implicated in estrogen-dependent cyclical uterine angiogenesis (Yasuda et al, 1998). In the central nervous system, expression of EPO receptors in neurons and brain capillaries is associated with a neuroprotective effect for EPO during ischemia-induced injury in vivo (Brines et al, 2000; Digicaylioglu and Lipton, 2001). The findings of our studies suggest that functional, nonhematopoietic EpoR expression can also be associated with the presence of a disease process, in this case breast cancer.

In normal mammary ducts surrounding tumor tissue, we did not observe EPO protein expression by immunohistochemistry, although EPO expression has been reported in lactating breast (Juul et al, 2000). To investigate for a possible relationship between tumor hypoxia and EPO immunoreactivity in tumor cells, we studied breast tumors labeled for tumor hypoxia by intravenous administration of pimonidazole hydrochloride to the patients, before tumor biopsies. Immunohistochemical analysis of contiguous tissue sections was carried out to detect colocalization of hypoxia and EPO expression in tumor cells. We did not find a consistent relationship between EPO expression and cellular hypoxia in our sample of tumors. Absence of colocalization of EPO and hypoxia immunoreactivity in a significant number of tumors is consistent with findings of an earlier study demonstrating lack of correlation between cellular hypoxia and expression of another hypoxia-inducible cytokine, vascular endothelial growth factor (Raleigh et al, 1998). Our data suggest that factors other than hypoxia in tumor cells may contribute to EPO expression in breast cancer cells. Transcriptional up-regulation of EPO mRNA expression in an estrogen-dependent manner in the female reproductive tract in the uterus (Yasuda et al, 1998) and oviduct (Masuda et al, 2000) suggests that estrogen may play a role in EPO expression in breast cancer cells. Our sample size was too small to establish a meaningful correlation between estrogen receptor status and EPO expression in the tumor specimens, and further analysis of a larger series will be required.
In many primary breast tumors, there was coexpression of EPO and EpoR protein, suggesting the potential for the generation of an autocrine or paracrine growth stimulatory loop, resulting in activation of EPO receptors that are expressed in breast cancer cells. Indeed, the presence of such an autocrine growthpromoting EPO-EpoR loop has been suggested in bone marrow progenitor cells, which were shown to express endogenous EPO (Sato et al, 2000; Stopka et al, 1998). In breast cancer cells that express prolactin receptor, another member of the type I cytokine receptor superfamily, autocrine secretion of prolactin was associated with increased proliferation (Yamauchi et al, 2000). A recent study by Acs et al (2001) reported EpoR expression in breast cancer and increased EPOinduced proliferation of breast cancer cell lines in vitro. Finally, expression of EPO and EpoR has been recently reported in renal carcinoma cells that also exhibited increased EPO-mediated proliferation (Westenfelder and Baranowski, 2000). Our data presented here and the results of these previous studies suggest functional significance for EPO and EpoR expression in cancer cells with respect to cancer cell proliferation.

We investigated the effect of EPO-EpoR antagonists on in vivo tumor growth using the T-ZC model. This method allows both local and systemic administration of putative anti-tumor compounds to assess their effects on tumor growth and angiogenesis in a reproducible manner. We found significant anti-tumor activity of three different antagonists of EPO-EpoR function in this system in a dose-dependent manner. The delay in tumor growth with an observed $45 \%$ reduction in maximum tumor depth in T-ZCs is remarkable, considering only a one-time administration of the antagonists at the time of implantation of the cancer cells in the animals. Thus, our data suggest that inhibitors of EpoR signaling may exhibit significant anti-tumor activity in vivo. Consistent with our results in breast cancer, the findings of a recent study by Yasuda et al (2001) suggested a functional role for EpoR signaling in cancers originating from the uterus and ovary in a xenograft model. Taken together, we propose that further studies of the role of functional EPO receptor expression in cancer cells may provide new insights into our understanding of cancer biology.

\section{Materials and Methods}

\section{Labeling of Tumor Hypoxia and Immunohistochemistry}

All human breast cancer specimens were obtained under a protocol approved by The Institutional Review Board at University of North Carolina Hospitals at Chapel Hill. Labeling of tumor hypoxia by intravenous pimonidazole hydrochloride administration to the patients was carried out as described previously (Raleigh et al, 1998) and multiple biopsies were obtained from distinct areas of primary breast tumors of each patient 24 hours after pimonidazole infusion. Fresh tissue was immediately placed in cold, $10 \%$ neutral buffered formalin and then processed into paraffin blocks. 
Before immunostaining, 4- $\mu \mathrm{m}$ sections from each block were placed on glass slides. A total of 26 biopsies from 10 primary breast cancer clinical specimens were studied and contiguous sections from two to three biopsy sites from each patient tumor were evaluated for EPO, EpoR expression, and the presence of pimonidazole adducts. Immunostaining for pimonidazole adducts using mouse monoclonal antisera $(1: 10,0)$ was carried out as described previously (Raleigh et al, 1998). Polyclonal, affinity purified, rabbit anti-human EPO receptor antibody (C-20) and polyclonal rabbit anti-EPO antibody $(\mathrm{H}-162)$ were from Santa Cruz Biotechnologies (Santa Cruz, California) and monoclonal anti-human EpoR antibody mh2er16.5.1 was generously provided by Genetics Institute (Cambridge, Massachusetts). The anti-EPO antibody and both anti-EpoR antibodies have been validated previously (Anagnostou et al, 1994; Brines et al, 2000; Fairchild Benyo and Conrad, 1999; Juul et al, 1998) and immunohistochemistry was carried out as described (Haroon et al, 2000). Briefly, anti-EpoR C-20 (1:50), anti-EpoR mh2er 16.5.1 (1:50), and anti-EPO (1:50) antibodies were hybridized overnight at $4^{\circ} \mathrm{C}$, followed by a series of incubations with secondary antibodies. Negative controls for the immunohistochemistry were treated with mouse or rabbit IgG instead of primary antibody and were negative in any reactivity.

\section{Tissue Culture, RNA, and Protein Analyses}

MCF-7, MDA-MB-231, and SK-BR3 human breast cancer cell lines were grown in DMEM with 10\% FCS and $1 \%$ penicillin-streptomycin (Life Technologies, Grand Island, New York). Total RNA from breast cancer cell lines was extracted using Qiagen RNAeasy kit (Qiagen, Studio City, California) according to the manufacturer's protocol. Total RNA isolated from a primary human breast carcinoma sample was purchased from Clontech (Palo Alto, California). All RNA samples were treated with DNase I (Life Technologies) before reverse transcription (RT) for cDNA synthesis, which was performed using oligo-dT priming and Superscript II according to manufacturer's protocol (Life Technologies). PCR amplification for EpoR was performed as described (Arcasoy et al, 1997), except the total number of amplification cycles was 40 and the extension step was 2 minutes for amplification of exons 1 through 8 of the entire EpoR coding region (Fig. 2A, Pcr3). Figure 2A illustrates the sizes and relative positions of expected PCR amplification products using three oligonucleotide primer pairs Pcr1, Pcr2, and Pcr3. The sense EpoR primers 5 ' $\rightarrow 3^{\prime}$ were: Exon 1: GCTGTATCATGGACCACCTC; Exon 5:GTGGAGATCCTGGAGGGCCG; and Exon 6: GCTGCTCTCCCACCGCCGGGCTCT. The antisense primers $5^{\prime} \rightarrow 3^{\prime}$ were all in exon 8: Pcr1: GCCAGAGTCAGATACCACAAG; Pcr2: GTCATATTGGATCCCTGATC; and Pcr3: CTGAGAGAGGCCTCGCCAT. Amplification products were separated in 1\% agarose gels and Southern blotting was performed according to standard protocols followed by hybridization to radioactively labeled 1.8-kb full-length human EpoR cDNA probe. The PCR products were subcloned into pCR4-TOPO vector (Life Technologies) and analyzed by automated sequencing at Duke University Medical Center sequencing facility.

\section{Immunoprecipitation and Immunoblotting}

Cells were grown to confluence in $10-\mathrm{cm}$ tissue culture plates and total cell lysates were prepared in ice-cold buffer containing 1\% Triton-X-100, $150 \mathrm{~mm}$ $\mathrm{NaCl}, 10 \%$ glycerol, $20 \mathrm{~mm}$ Tris- $\mathrm{HCl} \mathrm{pH}$ 7.4, 5 mm EDTA, $10 \mathrm{~mm} \mathrm{NaF}$ with protease inhibitors $1 \mathrm{~mm}$ PMSF, 10 $\mu \mathrm{g} / \mathrm{ml}$ leupeptin, $10 \mu \mathrm{g} / \mathrm{ml}$ pepstatin, $10 \mu \mathrm{g} / \mathrm{ml}$ aprotinin, and $1 \mathrm{mM} \mathrm{Na}_{3} \mathrm{VO}_{4}$. Immunoprecipitation was performed with $1 \mu \mathrm{g}$ anti-EpoR antibody $(\mathrm{C}-20)$ as described (Arcasoy et al, 2001), proteins were separated by $7.5 \%$ SDS-PAGE, and immunoblotting was carried out with C-20 antibody (1:200) and donkey anti-rabbit secondary antibody from Pierce (1:20,000). The proteins were detected using an ECL kit from Amersham-Pharmacia and subsequently subjected to autoradiography. As positive control, cell lysates of 32D cells expressing EpoR were used for immunoprecipitation.

\section{Animal Studies}

All animal protocols were approved by the Duke Institutional Animal Care and Use Committee. Fisher 344 rats of an average weight of $>150$ grams were purchased from Charles River Laboratories. The rats were kept in temperature-controlled rooms on a 12hour light-dark cycle with access to rodent chow and bottled tap water ad libitum. On the day of the experiments, the rats were anesthetized with intraperitoneal injections of sodium pentobarbital $(40 \mathrm{mg} / \mathrm{kg}$ ), the hair was removed using clippers, and the surface was surgically prepared. Two small midline skin incisions were made in the dorsal region approximately 4 $\mathrm{cm}$ apart. Fascia was blunt-dissected and small pockets were created on both sides along the midline incision to allow implantation of four T-ZCs per animal. T-ZCs are implanted in the subcutaneous tissue of rats and the incisions were closed with surgical clips and Neosporin applied to the surgical site. The T-ZC is a fibrin gel-based in vivo assay based on the concept developed by Dvorak et al (1987). These chambers are constructed from customized plexiglass rings with internal diameter of $10 \mathrm{~mm}$ and have an access port drilled on the side (Fig. 1G). The two open surfaces are covered by nylon mesh with 180 micron pore size (cat\# NY8H04700; Millipore Corporation, Bedford, Massachusetts) glued to the rings. Fibrinogen (cat\# 341578; Calbiochem, San Diego, California) is prepared in DMEM at a final concentration of $4 \mathrm{mg} / \mathrm{ml}$ and is converted to fibrin by addition of $8 \mu$ thrombin (100 $\mathrm{U} / \mathrm{ml}$; cat\#605160; Calbiochem) into the chambers (Haroon et al, 2002). These chambers allow the ingrowth of vessels, tumor histology closely resembles in situ conditions, and it has already been shown that early angiogenic response to human fibrinogen is very similar to rat fibrinogen in fibrin gel chambers (Rohr et al, 1992). In the T-ZC model, rat syngeneic R3230Ac 
mammary adenocarcinoma cells were suspended in fibrinogen solution $\left(2.5 \times 10^{6} \mathrm{cells} / \mathrm{chamber}\right)$ and 160 $\mu \mathrm{l}$ of the solution containing the test compound was injected into each chamber along with thrombin through the port. In these experiments, recombinant human soluble EPO receptor ( $R$ and $D$ Systems, Minneapolis, Minnesota) or neutralizing anti-EPO monoclonal antibody (MAB-287; R and D Systems) were reconstituted in PBS (cat\#14190-144, Life Technologies) and added to the chambers at the indicated final concentrations. Human sEpoR and neutralizing anti-EPO monoclonal antibody MAB-287 were capable of inhibiting murine EPO-mediated proliferation of hematopoietic cells expressing EpoR in vitro (unpublished data). Tyrphostin B42 (Calbiochem, AG 490) was reconstituted in DMSO immediately before administration into the T-ZC. Negative control groups consisted of T-ZCs with the addition of the same dilution of vehicle only (PBS or DMSO) and we found no difference in tumor growth among them. We performed these experiments in two sets. The controls were pooled for the final data set (22 of 24, 6 animals; and 16 of 16,4 animals, for a total of 38,10 animals) from two experiments. The number of treated T-ZCs were low dose (12 of 12, 3 animals), medium dose (14 of 16, 4 animals), and high dose (12 of 12,3 animals). All T-ZCs were harvested at Day 7 post-implantation. The tumor tissues were cut out from the chambers and preserved in $10 \%$ formalin for paraffin embedding. Tumor tissue sections were used after processing for hematoxylin and eosin (H\&E) staining and analysis of the maximum depth of tumor tissue inside the T-ZC to assess the degree of tumor growth. In this method treatment efficacy is assessed as percent growth from the controls thus the values are indicated as reduction from $100 \%$ (control). Two pathologists performed all measurements independently in a blinded fashion.

Statistical Analysis. GraphPad InStat version 3.00 software was used for all analyses. To determine significant effects of each treatment compared with controls, Students $t$ test was performed in each experiment.

\section{Acknowledgements}

The authors thank Ms. Lisa Licht and Dr. Cynthia Ballenger for assistance with patient recruitment, Genetics Institute for providing monoclonal anti-Epo receptor antibody, and Dr. Charles S. Greenberg for helpful discussions.

\section{References}

Acs G, Acs P, Beckwith SM, Pitts RL, Clements E, Wong K, and Verma A (2001). Erythropoietin and erythropoietin receptor expression in human cancer. Cancer Res 61:3561-3565.

Anagnostou A, Liu Z, Steiner M, Chin K, Lee ES, Kessimian $N$, and Noguchi CT (1994). Erythropoietin receptor mRNA expression in human endothelial cells. Proc Natl Acad Sci USA 91:3974-3978.
Arcasoy MO, Degar BA, Harris KW, and Forget BG (1997). Familial erythrocytosis associated with a short deletion in the erythropoietin receptor gene. Blood 89:4628-4635.

Arcasoy MO, Maun NA, Perez L, Forget BG, and Berliner N (2001). Erythropoietin mediates terminal granulocytic differentiation of committed myeloid cells with ectopic erythropoietin receptor expression. Eur J Haematol 67:77-87.

Bazan JF (1990). Structural design and molecular evolution of a cytokine receptor superfamily. Proc Natl Acad Sci USA 87:6934-6938.

Bright JJ, Du C, and Sriram S (1999). Tyrphostin B42 inhibits $\mathrm{IL}-12$-induced tyrosine phosphorylation and activation of Janus kinase-2 and prevents experimental allergic encephalomyelitis. J Immunol 162:6255-6262.

Brines ML, Ghezzi P, Keenan S, Agnello D, de Lanerolle NC, Cerami C, Itri LM, and Cerami A (2000). Erythropoietin crosses the blood-brain barrier to protect against experimental brain injury. Proc Natl Acad Sci USA 97:10526-10531.

Bunn HF and Poyton RO (1996). Oxygen sensing and molecular adaptation to hypoxia. Physiol Rev 76:839-885.

D'Andrea AD, Fasman GD, and Lodish HF (1990). A new hematopoietic growth factor receptor superfamily: Structural features and implications for signal transduction. Curr Opin Cell Biol 2:648-651.

D'Andrea AD, Lodish HF, and Wong GG (1989). Expression cloning of the murine erythropoietin receptor. Cell 57:277285.

Digicaylioglu M and Lipton SA (2001). Erythropoietinmediated neuroprotection involves cross-talk between Jak2 and NF-kappaB signalling cascades. Nature 412:641-647.

Dvorak HF, Harvey VS, Estrella P, Brown LF, McDonagh J, and Dvorak AM (1987). Fibrin containing gels induce angiogenesis. Implications for tumor stroma generation and wound healing. Lab Invest 57:673-686.

Fairchild Benyo D and Conrad KP (1999). Expression of the erythropoietin receptor by trophoblast cells in the human placenta. Biol Reprod 60:861-870.

Haroon ZA, Amin K, Saito W, Wilson W, Greenberg CS, and Dewhirst MW (2002). SU5416 delays wound healing through inhibition of TGF-beta 1 activation. Cancer Biol Ther 1:121126

Haroon ZA, Raleigh JA, Greenberg CS, and Dewhirst MW (2000). Early wound healing exhibits cytokine surge without evidence of hypoxia. Ann Surg 231:137-147.

Juul SE, Yachnis AT, and Christensen RD (1998). Tissue distribution of erythropoietin and erythropoietin receptor in the developing human fetus. Early Hum Dev 52:235-249.

Juul SE, Zhao Y, Dame JB, Du Y, Hutson AD, and Christensen RD (2000). Origin and fate of erythropoietin in human milk. Pediatr Res 48:660-667.

Klingmüller U (1997). The role of tyrosine phosphorylation in proliferation and maturation of erythroid progenitor cellssignals emanating from the erythropoietin receptor. Eur J Biochem 249:637-647.

Krantz SB (1991). Erythropoietin. Blood 77:419-434.

Lacombe C and Mayeux P (1998). Biology of erythropoietin. Haematologica 83:724-732. 
Lin CS, Lim SK, D'Agati V, and Costantini F (1996). Differential effects of an erythropoietin receptor gene disruption on primitive and definitive erythropoiesis. Genes Dev 10:154164.

Livnah O, Stura EA, Middleton SA, Johnson DL, Jolliffe LK, and Wilson IA (1999). Crystallographic evidence for preformed dimers of erythropoietin receptor before ligand activation. Science 283:987-990.

Lodish HF, Hilton DJ, Klingmuller U, Watowich SS, and Wu H (1995). The erythropoietin receptor: Biogenesis, dimerization, and intracellular signal transduction. Cold Spring Harb Symp Quant Biol 60:93-104.

Masuda S, Kobayashi T, Chikuma M, Nagao M, and Sasaki R (2000). The oviduct produces erythropoietin in an estrogenand oxygen-dependent manner. Am J Physiol Endocrinol Metab 278:E1038-1044.

Masuda S, Nagao M, Takahata K, Konishi Y, Gallyas F Jr, Tabira T, and Sasaki, R (1993). Functional erythropoietin receptor of the cells with neural characteristics. Comparison with receptor properties of erythroid cells. J Biol Chem 268:11208-11216.

Meydan N, Grunberger T, Dadi H, Shahar M, Arpaia E, Lapidot Z, Leeder JS, Freedman M, Cohen A, Gazit A, Levitzki A, and Roifman CM (1996). Inhibition of acute lymphoblastic leukaemia by a Jak-2 inhibitor. Nature 379:645648.

Morishita E, Masuda S, Nagao M, Yasuda Y, and Sasaki R (1997). Erythropoietin receptor is expressed in rat hippocampal and cerebral cortical neurons, and erythropoietin prevents in vitro glutamate-induced neuronal death. Neuroscience 76:105-116.

Nielsen M, Kaltoft K, Nordahl M, Ropke C, Geisler C, Mustelin T, Dobson P, Svejgaard A, and Odum N (1997). Constitutive activation of a slowly migrating isoform of Stat3 in mycosis fungoides: Tyrphostin AG490 inhibits Stat3 activation and growth of mycosis fungoides tumor cell lines. Proc Natl Acad Sci USA 94:6764-6769.

Ogilvie M, Yu X, Nicolas-Metral V, Pulido SM, Liu C, Ruegg UT, and Noguchi CT (2000). Erythropoietin stimulates proliferation and interferes with differentiation of myoblasts. J Biol Chem 275:39754-39761.

Raleigh JA, Calkins-Adams DP, Rinker LH, Ballenger CA, Weissler MC, Fowler WC Jr, Novotny DB, and Varia MA (1998). Hypoxia and vascular endothelial growth factor expression in human squamous cell carcinomas using pimonidazole as a hypoxia marker. Cancer Res 58:3765-3768.

Remy I, Wilson IA, and Michnick SW (1999). Erythropoietin receptor activation by a ligand-induced conformation change. Science 283:990-993.

Rohr S, Toti F, Brisson C, Albert A, Freund M, Meyer C, and Cazenave JP (1992). Quantitative image analysis of angiogenesis in rats implanted with a fibrin gel chamber. Nouv Rev Fr Hematol 34:287-294.

Sakanaka M, Wen TC, Matsuda S, Masuda S, Morishita E, Nagao M, and Sasaki R (1998). In vivo evidence that erythropoietin protects neurons from ischemic damage. Proc Natl Acad Sci USA 95:4635-4640.
Sato T, Maekawa T, Watanabe S, Tsuji K, and Nakahata T (2000). Erythroid progenitors differentiate and mature in response to endogenous erythropoietin. J Clin Invest 106: 263-270.

Stopka T, Zivny JH, Stopkova P, Prchal JF, and Prchal JT (1998). Human hematopoietic progenitors express erythropoietin. Blood 91:3766-3772.

Westenfelder C and Baranowski RL (2000). Erythropoietin stimulates proliferation of human renal carcinoma cells. Kidney Int 58:647-657.

Westenfelder C, Biddle DL, and Baranowski RL (1999). Human, rat, and mouse kidney cells express functional erythropoietin receptors. Kidney Int 55:808-820.

Witthuhn BA, Quelle FW, Silvennoinen O, Yi T, Tang B, Miura O, and Ihle JN (1993). JAK2 associates with the erythropoietin receptor and is tyrosine phosphorylated and activated following stimulation with erythropoietin. Cell 74:227-236.

Wojchowski DM, Gregory RC, Miller CP, Pandit AK, and Pircher TJ (1999). Signal transduction in the erythropoietin receptor system. Exp Cell Res 253:143-156.

Wu H, Lee SH, Gao J, Liu X, and Iruela-Arispe ML (1999). Inactivation of erythropoietin leads to defects in cardiac morphogenesis. Development 126:3597-3605.

Wu H, Liu X, Jaenisch R, and Lodish HF (1995). Generation of committed erythroid BFU-E and CFU-E progenitors does not require erythropoietin or the erythropoietin receptor. Cell 83:59-67.

Yamauchi T, Yamauchi N, Ueki K, Sugiyama T, Waki H, Miki H, Tobe K, Matsuda S, Tsushima T, Yamamoto T, Fujita T, Taketani Y, Fukayama M, Kimura S, and Yazaki Y (2000). Constitutive tyrosine phosphorylation of ErbB-2 via Jak2 by autocrine secretion of prolactin in human breast cancer. J Biol Chem 275:33937-33944.

Yasuda Y, Masudă S, Chikuma M, Inoue K, Nagao M, and Sasaki R (1998). Estrogen-dependent production of erythropoietin in uterus and its implication in uterine angiogenesis. J Biol Chem 273:25381-25387.

Yasuda $Y$, Musha T, Tanaka H, Fujita Y, Fujita $H$, Utsumi $H$, Matsuo T, Masuda S, Nagao M, Sasaki R, and Nakamura $Y$ (2001). Inhibition of erythropoietin signalling destroys xenografts of ovarian and uterine cancers in nude mice. $\mathrm{Br} J$ Cancer 84:836-843.

Youssoufian H, Longmore G, Neumann D, Yoshimura A, and Lodish HF (1993). Structure, function, and activation of the erythropoietin receptor. Blood 81:2223-2236.

Zhuang H, Niu Z, He TC, Patel SV, and Wojchowski DM (1995). Erythropoietin-dependent inhibition of apoptosis is supported by carboxyl-truncated receptor forms and blocked by dominant-negative forms of Jak2. J Biol Chem 270:14500-14504.

Zhuang H, Patel SV, He TC, Sonsteby SK, Niu Z, and Wojchowski DM (1994). Inhibition of erythropoietin-induced mitogenesis by a kinase-deficient form of Jak2. J Biol Chem 269:21411-21414. 